\section{Daily physical activity in subjects with newly diagnosed COPD}

Rationale Information about daily physical activity levels (PAL) in subjects with undiagnosed chronic obstructive pulmonary disease (COPD) is scarce. This study aims to assess PA and to investigate the associations between PA and clinical characteristics in subjects with newly diagnosed COPD.

Methods Fifty-nine subjects with a new spirometry-based diagnosis of mild $(n=38)$ and moderate $(n=21)$ COPD $(63 \pm 6$ years, $68 \%$ male) were matched with 65 smoking controls $(62 \pm 7$ years, $75 \%$ male). PA (daily steps, time spent in moderate-to-vigorous intense physical activities (MVPA) and PAL) was measured by accelerometry. Dyspnoea, complete pulmonary function tests, peripheral muscle strength and exercise capacity served as clinical characteristics.

Results PA was significantly lower in COPD versus smoking controls $(7986 \pm 2648$ vs 9765 \pm 3078 steps, 64 (27-120) vs 110 (55-164) min of MVPA, $1.49 \pm 0.21$ vs $1.62 \pm 0.24$ PAL respectively, all $p<0.05)$. Subjects with COPD with either mild symptoms of dyspnoea (mMRC 1), those with lower diffusion capacity $\left(T_{L}, C 0\right)$, low 6 min walking distance (6MWD) or low maximal oxygen uptake $\left(\mathrm{VO}_{2}\right.$ peak) had significantly lower PA. Multiple regression analysis identified $6 \mathrm{MWD}$ and $\mathrm{T}_{\mathrm{L}, \mathrm{CO}}$ as independent predictors of PA in COPD.

Conclusions The reduction in PA starts early in the disease, even when subjects are not yet diagnosed with COPD. Inactivity is more pronounced in subjects with mild symptoms of dyspnoea, lower levels of diffusion capacity and exercise capacity.
The detection of an inactive lifestyle in patients with chronic obstructive pulmonary disease (COPD) is increasingly important since inactivity predicts prognosis in $\mathrm{COPD}^{1}$ and may even impact on the rate of lung function decline. ${ }^{2}$ Data on daily physical activity levels (PAL) are lacking regarding patients unaware of their disease in whom the diagnosis of COPD is based on spirometry screening (preclinical stage). We aimed to objectively investigate daily PALs and to investigate the association between physical activity and different clinical characteristics in subjects with newly spirometry-based diagnosis of COPD.

Hundred and twenty-four (ex-) smokers were recruited from a population-based sample (see online supplementary figure S1). Fifty-nine subjects with a new spirometrybased diagnosis of mild $(n=38)$ and moderate $(\mathrm{n}=21)$ COPD $(63 \pm 6$ years, $68 \%$ male $)$ were matched with 65 smoking controls $(62$ \pm 7 years, $75 \%$ male). Detailed characteristics of the study subjects are summarised in online supplementary table S1. Physical activity (daily steps, time spent in moderate-to- vigorous intense physical activities (MVPA) and PAL) was measured by a multi-sensor activity monitor (SenseWear Pro 3 Armband). Dyspnoea, complete pulmonary function tests, peripheral muscle strength and exercise capacity served as clinical characteristics. Additional information on material and methods is available in the online supplementary.

We found that physical activity was significantly lower in COPD compared to smoking controls (figure 1). Subjects with COPD with either mild symptoms of dyspnoea (mMRC 1), those with lower diffusion capacity $\left(\mathrm{T}_{\mathrm{L}}, \mathrm{co}\right)$, low $6 \mathrm{~min}$ walking distance $(6 \mathrm{MWD})$ or low maximal oxygen uptake $\left(\mathrm{VO}_{2}\right.$ peak $)$ had significantly lower PALs (see online supplementary figure S2-S5). COPD subjects and smoking controls with lower levels of isometric quadriceps force did not show lower daily PALs. Multiple regression analysis identified 6MWD and $T_{L}$, co as independent predictors of physical activity in COPD (see online supplementary table S2).

Our data support the recent advice of Centers for Disease Control and Prevention that physical activity is an important vital sign, even in patients with mild disease. ${ }^{3}$

Several cross-sectional studies found that patients with an established diagnosis of mild-to-moderate COPD, recruited in hospital outpatient settings, were physically inactive compared to a (non-) smoking control group. ${ }^{45}$ This is the first study that showed that early reduction in physical activity is already present in subjects with mild-to-moderate COPD who did not previously present to healthcare services (ie, preclinical stage). Of importance to clinicians is the finding that some clinical characteristics (mild symptoms of dyspnoea, low values of diffusion capacity and exercise capacity) may identify the inactive subjects. In this group, early therapeutic interventions such as activity counselling programmes could be helpful in preventing deterioration of the PALs, and by consequence, other clinical outcomes such as comorbidity and disease progression. ${ }^{2}$ We conclude that the reduction in physical activity starts early in the disease, even when subjects are not yet diagnosed with COPD, especially in those
A

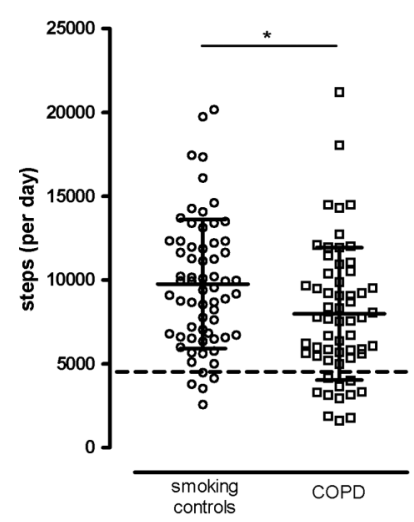

B

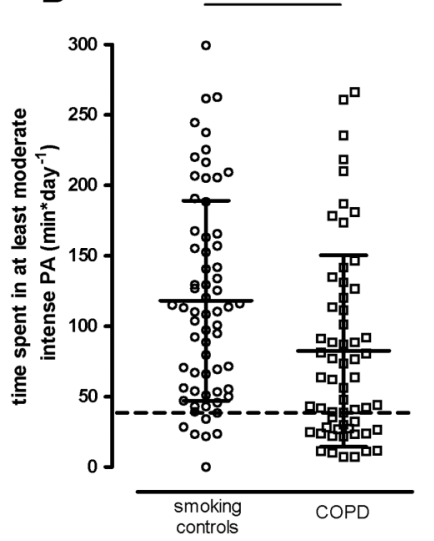

C

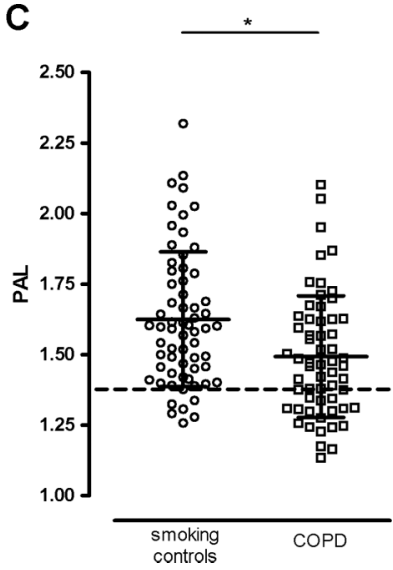

Figure 1 Daily physical activity levels (PAL) in subjects with and without chronic obstructive pulmonary disease (COPD); daily steps (A) 7986 \pm 2648 vs $9765 \pm 3078$ steps, daily time spent in moderate-to-vigorous physical activity (MVPA) (B) 64 (27-120) vs 110 (55-164) min of MVPA and daily PAL (C): $1.49 \pm 0.21$ vs $1.62 \pm 0.24$ PAL. * $p<0.05$ COPD versus smoking controls. 
with mild symptoms of dyspnoea, lower levels of diffusion capacity and exercise capacity.

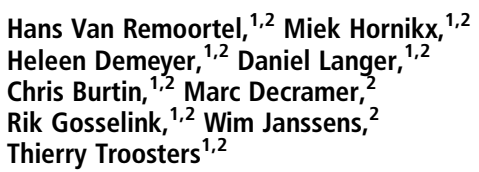

${ }^{1}$ Faculty of Kinesiology and Rehabilitation Sciences, Department of Rehabilitation Sciences, KU Leuven, Leuven, Belgium

${ }^{2}$ Respiratory Division and Rehabilitation, UZ Gasthuisberg, Leuven, Belgium

Correspondence to Dr Thierry Troosters, Respiratory Rehabilitation and Respiratory Division, UZ Gasthuisberg, Herestraat 49 bus 706, Onderwijs \& Navorsing I, Labo Pneumologie, Leuven B-3000, Belgium; thierry.troosters@med.kuleuven.be HVR and MH are contributed equally.

Acknowledgements The authors would like to thank Geert Celis and co-workers (Respiratory Division, E502, University Hospital Gasthuisberg, Leuven, Belgium) for their assistance with the lung function tests. Kristien De Bent, Laurence Vrancken, Erica Balligand, Claudia Carremans and Laura Jacobs (Clinical Trial Unit, University Hospital Gasthuisberg, Leuven, Belgium) are acknowledged for their assistance in patient recruitment and data collection. Kristof Van Eyken is acknowledged for his help in the data collection by creating a database. The Nelson team (department of Thoracic Surgery, UZ Gasthuisberg, Leuven, Belgium) is acknowledged for providing patient contacts.

Contributors HVR contributed to the protocol development, collected the data, performed data analysis and wrote the manuscript. MH contributed to the protocol development, collected the data, performed data analysis and wrote the manuscript. HD contributed to the statistical analysis, assisted in the data collection and critically reviewed the manuscript. $\mathrm{DL}$ contributed to the protocol development, assisted in the data collection and critically reviewed the manuscript. $\mathrm{CB}$, DL contributed to the protocol development, assisted in the data collection and critically reviewed the manuscript. MD contributed to the protocol development and critically reviewed the manuscript. RG contributed to the protocol development and critically reviewed the manuscript. WJ provided the study idea, contributed to the protocol development and critically reviewed the manuscript. TT provided the study idea, contributed to the protocol development and critically reviewed the manuscript.

Funding This work was supported by Research Funds Flanders (G05.98.09N)

\section{Competing interests None.}

Ethics approval The study was approved by the local ethics committee (Medical Ethical Board of the University Hospitals Leuven, Belgium, approval number B32220096387).

Provenance and peer review Not commissioned; internally peer reviewed.

- Additional material is published online only. To view please visit the journal online (http://dx.doi.org/10.

1136/thoraxjnl-2013-203534).

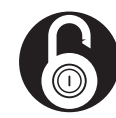

\section{OPEN ACCESS} distributed in accordance with the Creative Commons Attribution Non Commercial (CC BY-NC 3.0) license, which permits others to distribute, remix, adapt, build upon this work non-commercially, and license their derivative works on different terms, provided the original work is properly cited and the use is noncommercial. See: http://creativecommons.org/licenses/ by-nc/3.0/

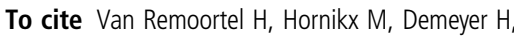
et al. Thorax 2013:68:962-963.

Received 8 March 2013

Accepted 22 March 2013

Published Online First 20 April 2013

Thorax 2013:68:962-963.

doi:10.1136/thoraxjnl-2013-203534

\section{REFERENCES}

1 Garcia-Aymerich J, Lange P, Benet M, et al. Regular physical activity reduces hospital admission and mortality in chronic obstructive pulmonary disease: a population based cohort study. Thorax 2006;61:772-8

2 Garcia-Aymerich J, Lange P, Benet M, et al. Regular physical activity modifies smoking-related lung function decline and reduces risk of chronic obstructive pulmonary disease: a population-based cohort study. Am J Respir Crit Care Med 2007;175: 458-63.

3 National physical activity plan of the United States of America. Health care, strategy 1 'Make physical activity a patient "vital sign" that all health care providers assess and discuss with their patients'. 2010 http://www.physicalactivityplan.org/ NationalPhysicalActivityPlan.pdf (accessed 25 Feb 2013)

4 Troosters T, Sciurba F, Battaglia S, et al. Physical inactivity in patients with COPD, a controlled multi-center pilot-study. Respir Med 2010;104:1005-11.

5 Watz H, Waschki B, Meyer T, et al. Physical activity in patients with COPD. Eur Respir J 2009;33: 262-72. 